\title{
Le fortificazioni costiere austroungariche sulla frontiera italiana nell'Istria e Dalmazia dagli studi dello Scacchiere orientale
}

\begin{abstract}
Austro-Hungarian coastal fortifications on the Italian border at Istria and Dalmatia from the studies on the eastern area
\end{abstract}

\section{Sara Isgrò}

Dipartimento di Architettura - Università degli Studi di Napoli Federico II, Naples, Italy, isgrosara@gmail.com

\begin{abstract}
Right after the Unification of Italy, land's topography, with landscape acquisition and restitution through explorations across borders, and in particular regarding Austro-Hungarian fortification on the Italian land and sea border, were immediately observed by Major State's officials. In early 1900 the long and jagged stretch of Dalmatian coast between Pola and Cattaro, full of natural ports and coastal canals formed by many islands sometimes arranged in multiple orders along the coast, and in the past defended by many works which are now mostly radiated or abandoned (except for S. Nicolò fort, near Sebenico), can count on some works realized in Lussinpiccolo (Monte Asino): Ragusa wall has been entirely unarmed and defensive organization of Cattaro's cannons is only maritime, in fact, for the part towards the land the Austrians provided to organize the defensive arming against neighboring Montenegro; Pola maritime square instead includes a sea front and a land front, so it can obtain protection by gulf, city's arsenal and Fasana Canal. Archive's material consulted in Kriegsarchiv of Vienna, historical cartography of AustroHungarian fortification system detected by Italian officers during Major State journeys on a side, together with many Memories on Austrian maritime fortifications between Cattaro and Pola, published by Major State Command, operations Division, allow to investigate and deepen Austro-Hungarian forts system along Italian coast, in Istria and Dalmatia.
\end{abstract}

Keywords: Fortifications, Istria, Dalmatia, Kotor, Pula.

\section{Premessa}

Sulla soglia del XX secolo, quando tutto o quasi accennava a nuove trasformazioni e a probabili progressi che sarebbero stati attuati in un avvenire non lontano nelle marine da guerra e che avrebbero modificato radicalmente le forme delle opere costiere e in special modo la composizione del loro armamento, il barone Ernesto Von Leithner, noto autore dei Principi fondamentali della fortificazione permanente moderna (Von Leithner), pubblicò uno studio sullo stato della fortificazione costiera allo scopo di esaminarne i tipi principali per constatare se rispondessero alle mutate esigenze, determinate essenzialmente dalla posizione e dallo scopo che le varie opere si proponevano, dai progressi registrati nella costruzione delle navi e dalla grande potenza delle artiglierie con cui queste ultime erano armate.

Era un momento delicatissimo, in cui la protezione fornita sino ad allora dalla corazzatura non poteva considerarsi completa, giacché le piastre di spessore ordinario venivano perforate dalle artiglierie moderne e i vantaggi offerti dalle nuove piastre d'acciaio con nichelio e sistema Harvey 
erano impiegate per diminuire il peso dalle corazze anziché per aumentare la loro resistenza, in ragione anche del notevole costo.

Mentre nelle fortificazioni di terra il ridotto centrale veniva protetto contro il bombardamento, stabilendo la linea di cinta in modo che fosse possibile impedire al nemico di collocarsi a distanza di tiro utile a colpire lo spazio da proteggersi, nelle fortificazioni di costa ciò non era sempre possibile, giacché spesso il posto da difendere e i suoi depositi si trovavano in una posizione sul mare che non permetteva la costruzione di opere molto avanzate. In tali casi l'unica soluzione possibile era quella di armare le opere con bocche da fuoco così potenti da impedire alle navi d'iniziare il bombardamento.

È interessante rilevare come i progressi nelle fortificazioni delle coste, in ragione dell'impiego di costruzioni corazzate, furono più celeri di quelli registrati nella fortificazione terrestre.

In generale, le regole stabilite per la fortificazione permanente di terra valevano anche per quella costiera, e andavano modificate in relazione alle bocche da fuoco più potenti a tiro di lancio a cui le fortificazioni da costa dovevano resistere e alla mancanza di obici e mortai nell'armamento dell'attaccante (Fortificazione delle coste, 1894).
Come nella progettazione della fortificazione terrestre, il disegno dei forti fu adattato, di volta in volta, alle diverse orografie del sito, tuttavia una diversità può ravvisarsi nello studio delle diverse soluzioni dell'organizzazione generale della fortificazione costiera, in ragione all'orografia delle coste, in questo caso più pregnante che non nello studio della fortificazione terrestre; furono infatti analizzati e descritti nel dettaglio, nella manualistica dell'epoca, i diversi casi così come le possibili soluzioni tecniche, costruttive e sopratutto logistiche da adottarsi di volta in volta.

\section{Introduzione}

La costa istriana, da Grado in poi, offriva alla Marina austro-ungarica un certo numero di porti e di ancoraggi sicuri, anche per navi da battaglia di grandi dimensioni, oltre a svariati ridossi naturali, rifugi temporanei per navi di minore portata e canali insulari costieri che permettevano alle imbarcazioni di spostarsi dall'una all'altra base al riparo dal nemico e che potevano essere interdetti con poche mine a ogni esplorazione; la costa italiana dell'epoca, non presentava nessun porto naturale in grado di rivaleggiare, anche lontanamente, con il lungo e frastagliato tratto di costa dalmata, con le insenature di Pola e Cattaro difeso da numerose opere che sorgevano a Lussinpic-

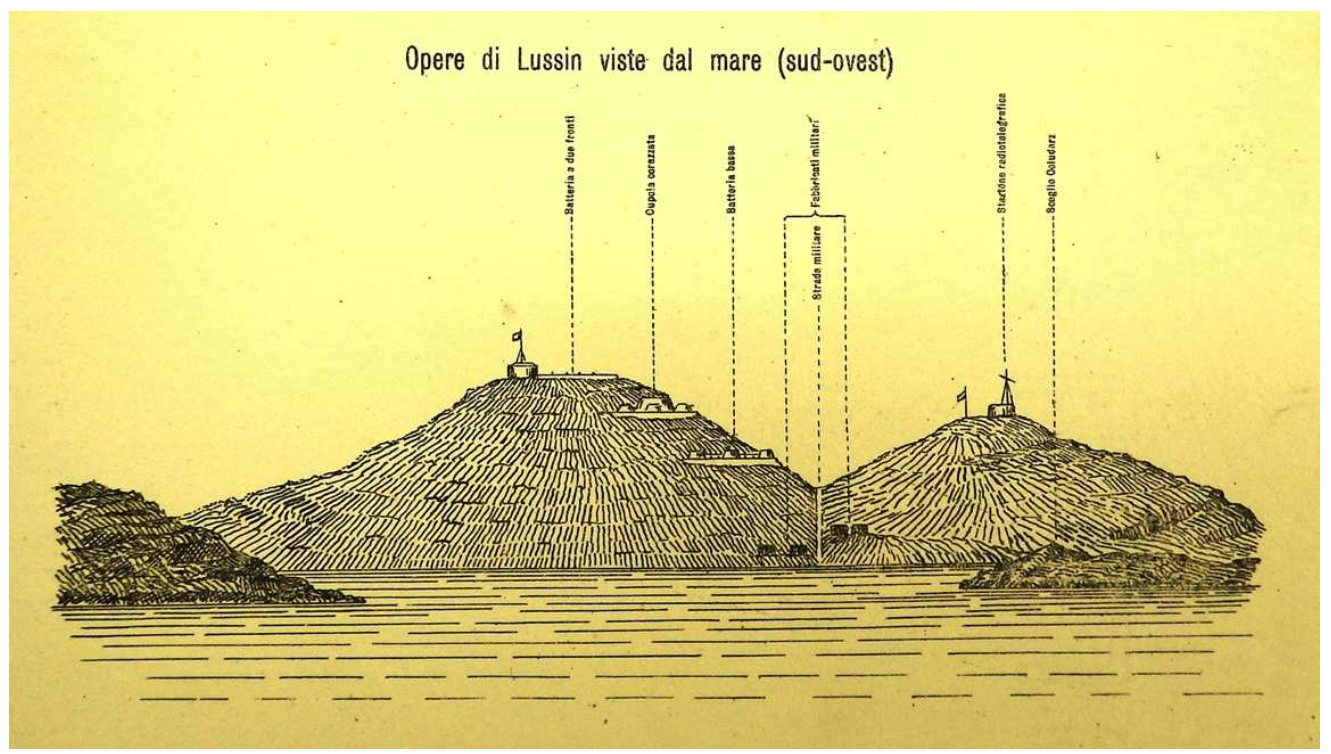

Fig. 1. Opera di Lussin vista dal mare (AUSSME). 


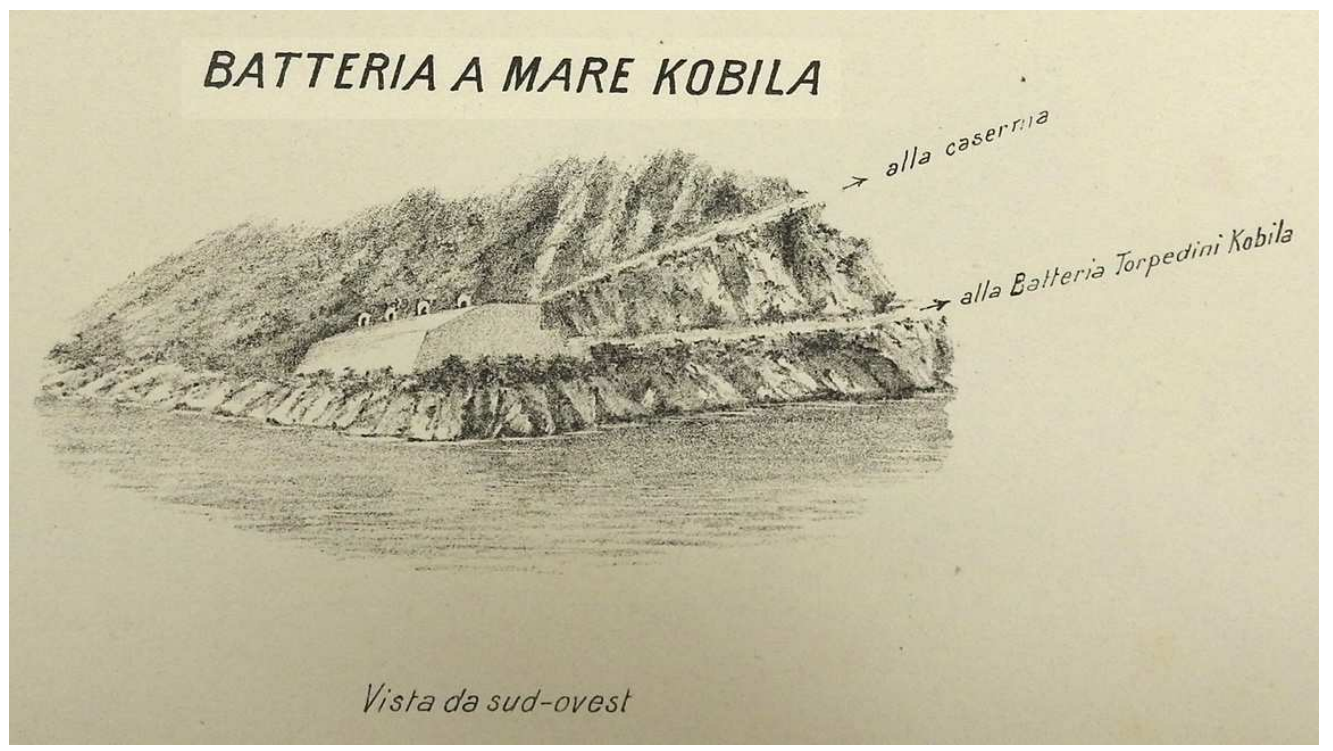

Fig. 2. Batteria a mare Kobila (AUSSME).

colo, Zara, Sebenico, Traù, Spalato, Clissa, Knin, Lissa, Gravosa e Ragusa. Opere che successivamente furono perlopiù radiate, abbandonate o utilizzate come caserme e magazzini (Bravetta, 1925).

Nel 1859, l'Austria pensò di rendere Sebenico, tra i porti naturali più vasti e sicuri dell'Adriatico, con funzione di collegamento tra i due punti di appoggio fortemente organizzati dalla flotta austriaca, Pola e Cattaro, il luogo principale di raccolta della marina da guerra, ma la ristrettezza dell'imbocco (costituito dal canale di S. Antonio), le difficoltà dell'ingresso con vento contrario e la poca profondità della baia della Maddalena, dove sarebbe dovuto sorgere l'arsenale, ne sconsigliarono la scelta come porto militare di prim'ordine.

L'esistenza del Forte di S. Niccolò, a protezione dell'imbocco del canale di S. Antonio, e l'intenzione manifesta dell'Austria di ostruire il canale stesso in caso di guerra con difese subacquee, dimostrano il proposito di proteggere il porto di Sebenico da sorprese e da operazioni di guerra, per farne località di rifugio per navi minori e torpediniere, oltre che deposito di carbone per la flotta da estrarre presso le miniere di Monte Promina sopra Dernis e da Dubravice presso Scadorna.

La zona tra Gravosa e Ragusa era costellata da numerose opere erette al fine di ostacolare l'azione marittima e terrestre contro le suddette città; oggi, solo il Forte Imperiale e la cinta di Ragusa si trovano in discrete condizioni.

\section{Importanza militare delle bocche di Cattaro}

Le bocche di Cattaro, per via della loro condizione geografica, hanno mantenuto nel tempo un ruolo di porto naturale di prim' ordine nel dominio dell' Adriatico stesso.

Per il naturale stato di isolamento che ha contraddistinto la zona rispetto alle linee di comunicazione terrestre, le bocche di Cattaro non sono mai state un porto militare rilevante, atto non solo al rifugio, ma anche al rifornimento delle flotte; mansioni per le quali era necessaria una maggiore vicinanza ai centri di deposito. Tuttavia, risultavano utili all'Austria come zona di raccolta della flotta militare, così come attestato dalla condizione del porto di Teodo, sia in pace sia in guerra.

L'Austria per la parte terrestre provvide a un ristretto ordinamento difensivo, limitandosi a provvedere ad un sostanziale sbarramento delle comunicazioni con il vicino Montenegro.

Per quanto attiene invece all'organizzazione difensiva delle bocche di Cattaro a livello marittimo, si provvide con la disposizione di opere, in grado di opporsi alle azioni delle flotte nemiche 
tendenti a penetrare nell'interno delle bocche, e con opere che ne ostacolassero gli sbarchi a sud e a nord delle bocche verso le fortificazioni costiere.

In definitiva, il progetto difensivo delle bocche di Cattaro si articolava in due gruppi di opere: sistemazione difensiva del mare; sistemazione difensiva contro il Montenegro.

Il sistema difensivo delle bocche da mare, nella fattispecie, sfruttò la speciale e naturale configurazione delle bocche stesse, munite di baie e stretti canali, così da collocare le opere nelle aree dei restringimenti, utili per lo sviluppo di azioni convergenti.

Le linee di difesa da mare erano due, la prima collocata tra Punta d'Ostro e Punta d'Arza, la seconda più interna, costituita da opere di più recente costruzione, disposte in due gruppi: uno sulla penisola di Lustica (dove sorgeva anche l'opera Liumantics, ex blockhaus Lustica) e l'altro su quella di Kobila.

Entrambi erano organizzati e costruiti secondo lo stesso impianto, comprensivo di due opere principali (Forte Kaballa e Forte Lustica, Batteria Kobila superiore e inferiore), di una batteria secondaria (Batteria Kaballa, Batteria a mare Kobila) e di una batteria di torpedini munita anche di caserme e fabbricati di carattere militare.

Nella parte interna delle bocche non si erano apprestati altri sistemi difensivi, ma solo uno sbarramento a Kumbur, un'ostruzione alle "Catene" protetta da altre costruzioni e, sopra Castelnuovo, un rimodernamento dell'armamento dell' antico Forte Spagnuolo.

Nella zona della baia di Traste, per impedire lo sbarco di truppe nemiche che avrebbero attaccato sul rovescio le opere del fronte mare o bombardato la zona di Toedo, furono erette due opere fondamentali: Forte Radisevic e Forte Grabovac sull'istmo di Krtole.

\section{Piazzaforte marittima di Pola}

Dopo l'intermezzo francese degli anni 18051813, sotto l'Imperatore Francesco Giuseppe I cominciò un periodo di stabilizzazione e consolidamento che portò Pola a diventare un vero centro industriale fortificato. Con il Piano generale di difesa dell'Impero del 1827 il governo austriaco scelse il porto di Pola come base della marina da guerra e, attorno al 1830, iniziò la costruzione delle prime fortificazioni circolari a casamatta.

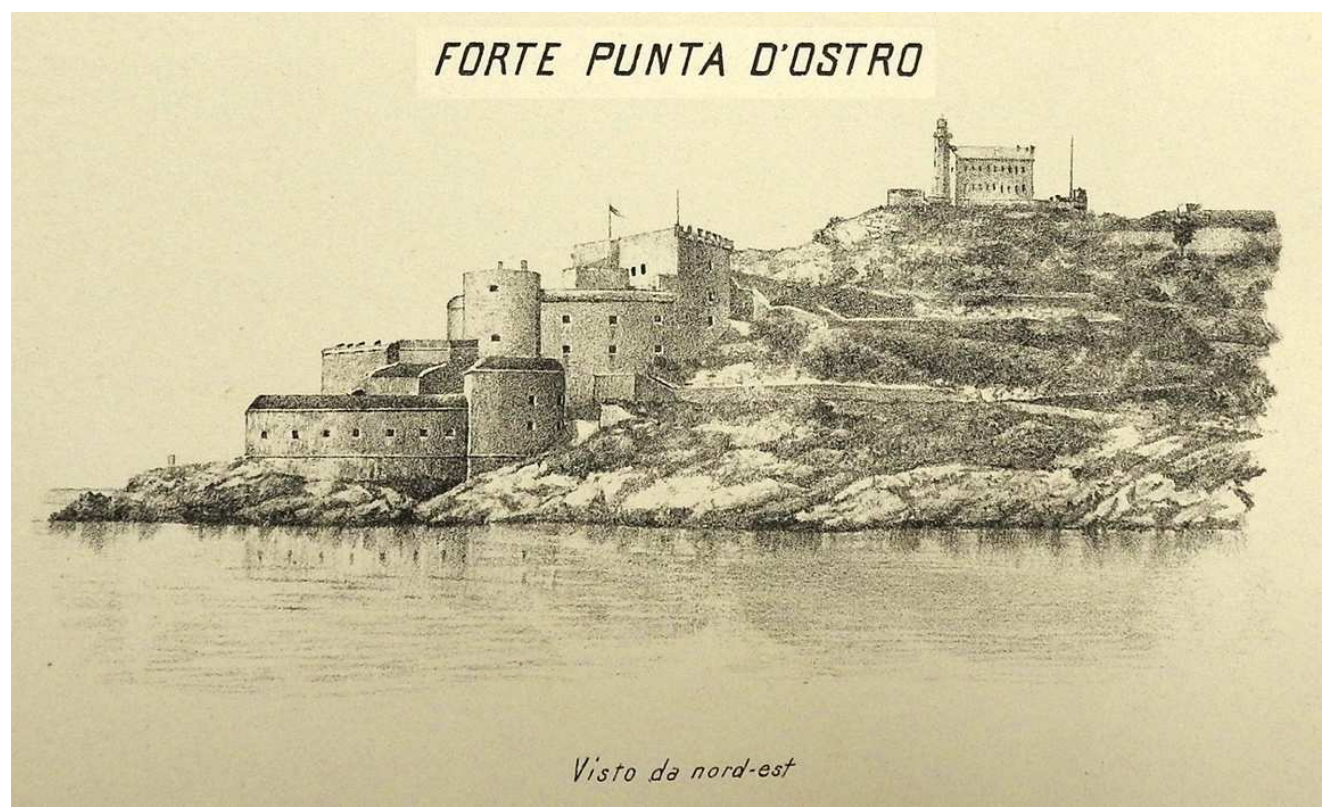

Fig. 3. Forte punta d'Ostro (AUSSME). 
Dal 1832 al 1859, al fine di permettere una più efficace difesa del golfo di Pola, vennero costruite tre torri d'artiglieria del diametro di $24 \mathrm{~m}$, oltre ad altre tre torri circolari minori. La prima torre d'artiglieria era il Forte Massimiliano, la seconda si trovava sull'isola di Sant'Andrea e la terza a Punta Cristo, inglobata nelle successive fasi di ampliamento del forte. Successivamente tra 1850 e 1875 venne intensificata la costruzione di forti e batterie d'artiglieria adeguate ai nuovi tipi di armamento e agli sviluppi delle tecniche militari.

Furono quindi realizzate nuove fortificazioni dall'impianto circolare con un diametro variabile dai 30 ai $110 \mathrm{~m}$, denominate torri polesi, a cui si aggiunse come variante la torre polese segmentata.

Giacché le opere poste in posizione elevate sul livello del mare poco o nulla avevano a temere del tiro delle navi, e le opere basse dovevano premunirsi soltanto contro i colpi diretti e di lancio, non di certo contro il tiro arcato da mare, sfruttando l'orografia del terreno e seguendo una logica di collocazione delle fortificazioni in cima ai colli e nei punti strategici fu realizzata una corona di fortezze e batterie d'artiglieria a diversa distanza dalla città.

A partire dal 1885, in risposta all'aumento del potenziale offensivo rappresentato dalle nuovi armi d'artiglieria, a Pola e nel suo circondario furono eseguite nuove e importanti realizzazioni militari, sino a giungere a integrarsi in tre continue linee fortificate verso il fronte di terra che, insieme alle zone di mare minate, alle ostruzioni retali e alle difese costiere, trasformarono Pola in una vera città fortificata.

Da allora e fino alla Prima guerra mondiale, il sistema difensivo fu radicalmente riorganizzato. Le precedenti fortificazioni circolari lasciarono il passo alle nuove possenti fortificazioni poligonali dotate di artiglierie di maggiore gittata e di maggiore calibro. Alcuni di questi forti, come il forte Brioni Minor, erano delle cittadelle scavate nella roccia, da cui affioravano cupole blindate d'acciaio in cui erano sistemati i cannoni.

\subsection{L'organizzazione difensiva}

Durante la Prima guerra mondiale l'organizzazione difensiva della piazzaforte di Pola era divisa in XVI Distretti di Difesa a cui corrispondevano una o più opere, e ogni distretto poteva ancora suddividersi in Punti d'appoggio.

Le opere erano così raggruppate:

1. Gruppo avanzato di Barbariga: sorgeva nelle vicinanze di Punta Barbariga allo scopo di difendere l'entrata nord-ovest del Canale di Fasana;

2. Gruppo delle isole Brioni: costruito sulle omonime isole, era destinato alla protezione del Canale di Fasana; batteva il mare ed entrambe gli ingressi al canale, ed era diviso in 3 sottogruppi:

a. $1^{\circ}$ sottogruppo dell'Isola Brioni Minore: Forte Brioni Minore, Batteria Brioni Minore o Batteria S. Niccolò, batteria di spiaggia;

b. $2^{\circ}$ sottogruppo: Forte Tegethoff;

c. $3^{\circ}$ sottogruppo, detto Peneda: Forte Franz Joseph o Forte Peneda e Forte Cavarolla entrambe con batteria in spiaggia, Batteria Naviglio.

3. Gruppo di Pola: il fronte a mare si estendeva da Punta Verudella a Punta Compare e nelle vicinanze di Punta Cristo e Stignano; aveva azione sull'alto mare, chiudeva l'imbocco del Canale di Fasana e bloccava l'entrata del porto di Pola. Era formato da quattro sottogruppi:

a. Sottogruppo nord-est: Forte Cristo e batterie di spiaggia, Batteria Maestà, Batteria di Valmaggiore o Punta Grossa, Batteria di Monte Grosso, Batteria Coseda sull'isolotto omonimo;

b. Sottogruppo di sbarramento: Forte Franz, batteria di spiaggia di S. Caterina;

c. Sottogruppo Mussil: Forte Maria Luisa, Batteria Signole, Batteria Fisella, Forte Mussil, Batteria Ovina, Batteria Max o di Monte Orlando, Forte Stoja di Mussil con batteria da spiaggia;

d. Sottogruppo Verudella: Batteria Monsival, Batteria S. Giovanni Verudella, Forte Verudella, Batteria mortai Verudella, Batteria intermedia Verudella, Batteria Pomer, Batteria Monte Guerra. 


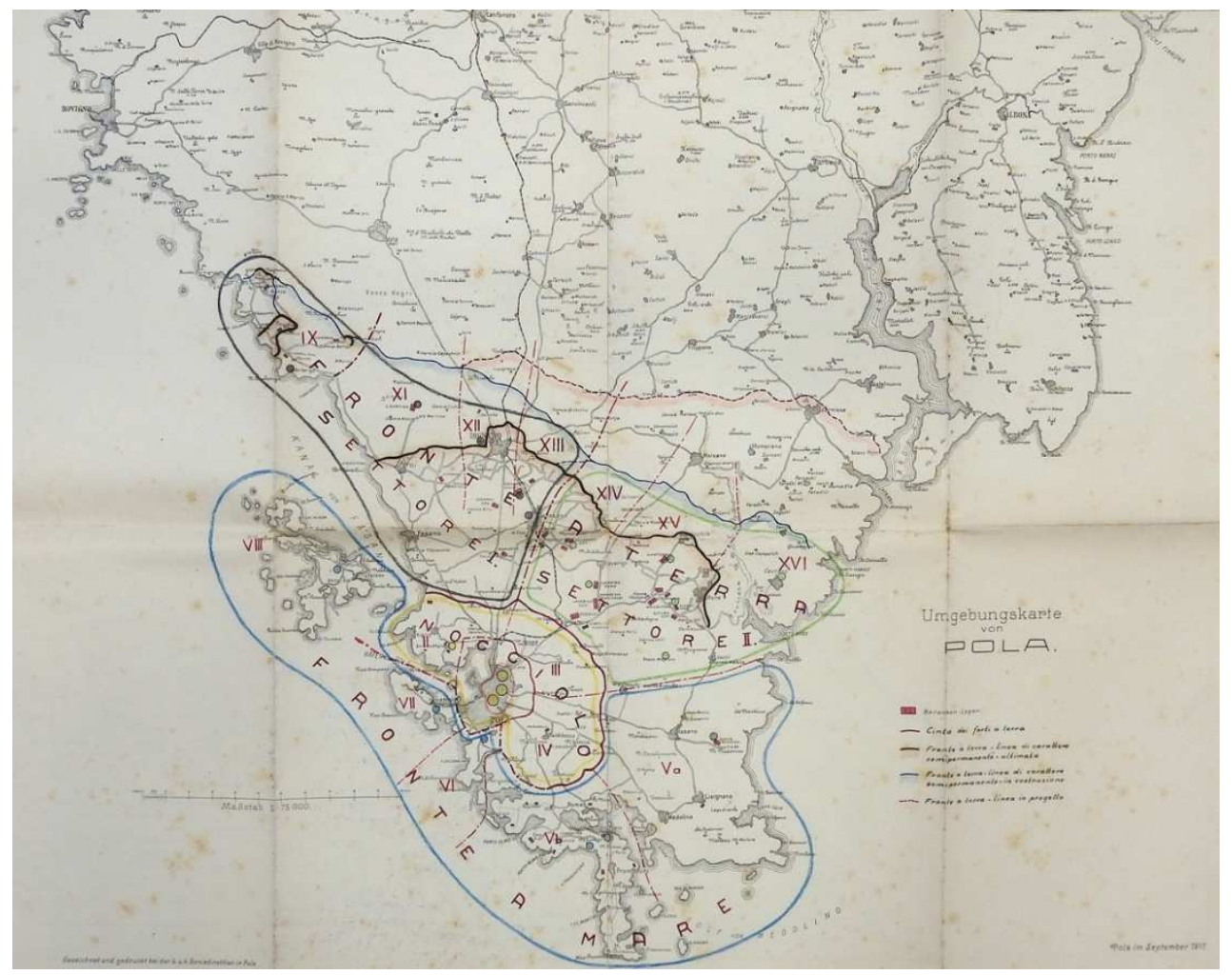

Fig. 4. Studio del fronte di terra. Individuazione dei XVI Distretti (ISCAG, 1918).

Il forte a terra era invece costituito da una doppia linea di forti che presidiavano gli arrivi e gli attacchi dall'interno. La linea più vicina alla piazza comprendeva cinque forti, Pomer, Turcian, S. Daniele, Bradamante e Castellier, e una batteria di obici tra i forti Bradamante e S. Daniele.

L'organizzazione difensiva della piazza comprendeva inoltre costruzioni a uso caserma, laboratori, magazzini, ecc., necessari per il buon funzionamento delle iniziative difensive; nonché stazioni radiotelegrafiche, telegrafiche, telefoniche, fotoelettriche, strade militari, sbarramenti e ogni tipo di ostruzione.

In prospettiva, quello vissuto da Pola durante il periodo austro-ungarico oggi può giustamente considerarsi un momento di rinascita e prosperità, sviluppo legato alla sua valenza militare come principale porto da guerra della marina asburgica, così come non è errato valutare come un lungo periodo di declino quello seguito alla dissolu- zione dell'Austro-Ungheria e al passaggio al Regno d'Italia, segnatamente marcato da un ridimensionamento della sua rilevanza militare per cui furono, se non sospese, drasticamente ridotte tutte le attività legate agli armamenti e all'equipaggiamento navale.

\section{Conclusione}

Le architetture fortificate sorte a difesa e controllo militare di un intero territorio, tra cui le fortificazioni costiere, nel tempo hanno stretto con esso un inscindibile rapporto, nonostante le trasformazioni, gli adattamenti, gli abbandoni, talvolta anche le distruzioni, concorrendo a segnare i caratteri identitari dei luoghi e delle singole comunità che in questa strutturazione riconoscono la propria storia.

Indubbiamente, per Pola fu proprio l'intero apparato difensivo a scoraggiare qualsiasi azione offensiva con attacchi da terra o da mare ed è forse 
grazie a ciò che oggi la città dispone di un complesso fortificatorio ben conservato, costruito per lo più sui belvedere dai quali si gode ancora un panorama di rara bellezza.

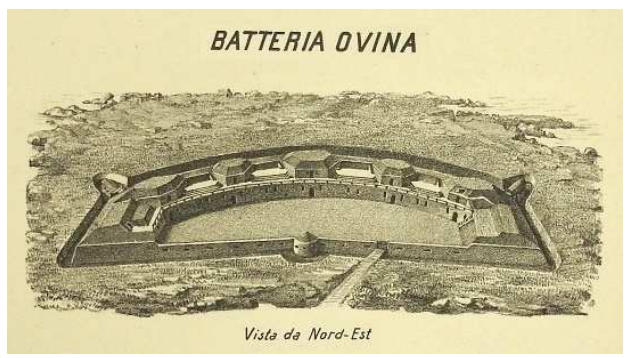

Fig. 5. Batteria Ovina (AUSSME).

$\mathrm{Al}$ riguardo, vale ciò che più volte è stato sottolineato da Giovanni Carbonara, non da ultimo in occasione delle Giornate di studio sull'Architet- tura Fortificata tenutesi a Bari nel 2006, che sottolineando la validità dell'unità concettuale e metodologica nel campo del restauro, ha osservato come non si debbano eccettuare le architetture fortificate dal patrimonio architettonico in generale, ponendo l'accento sui diversi fattori di rischio cui tale patrimonio difficile, per usare le parole di Claudio Varagnoli, è esposto, se non altro per avere perso l'originaria destinazione d'uso, ma che nel contempo apre ad un interessante rapporto con l'architettura contemporanea.

Oltre a garantire un riuso consono al valore delle fabbriche, in ragione delle problematiche legate anche all'accessibilità di tale patrimonio, occorre trovare un nuovo rapporto con l'intorno capace di stabilire una nuova reciprocità tra le parti, facendo dunque ricorso a una progettazione a più livelli, che oltre a tenere conto degli aspetti prettamente conservativi e/o restaurativi affronti i temi

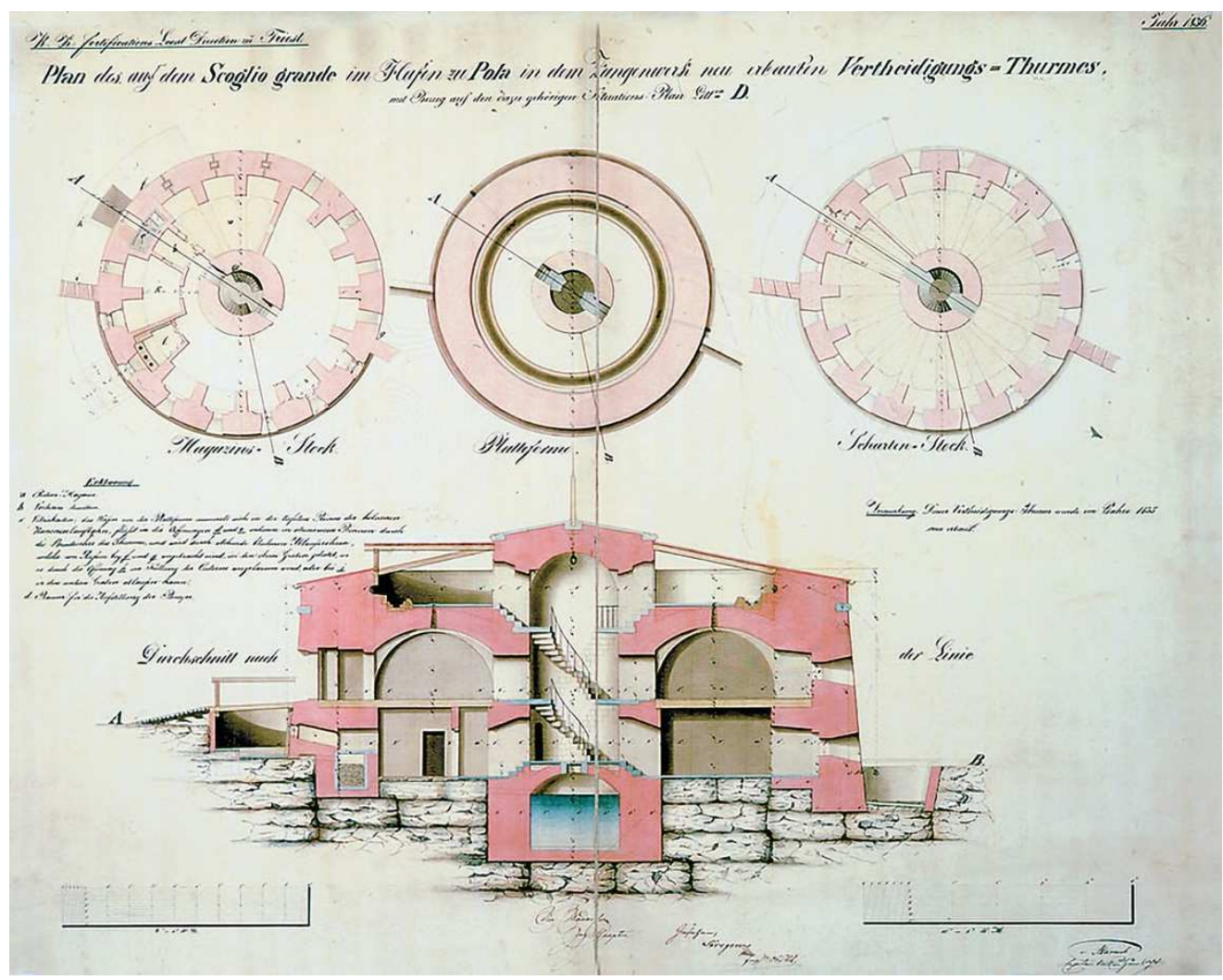

Fig. 6. Forte San Giorgio a Pola (Kriegsarchiy, Vienna). 
della fruizione e del riuso, con scelte mature in grado di condurre alla definizione dell'alternativa più sostenibile fra conservazione e fruizione.

Diverse le iconografie relative alle fortificazioni costiere austroungariche nell'Istria e Dalmazia conservate al Kriegsarchiv di Vienna e messe gentilmente a disposizioni ai fini del presente studio dal Museo Storico Italiano della Guerra di Rovereto, ma il tema è di una portata tale che non può di certo affrontarsi in quest'occasione meno che mai esaurirsi nel numero di battute previste dalla Call di questo convegno, senza rischiare di apparire superficiali. Ciò ha motivato la scelta di fare ricorso in questa sede ad alcune iconografie, pur sempre inedite, conservate all'Archivio dell'Ufficio Storico dello Stato Maggiore dell'Esercito (AUSSME) o all'Istituto di Storia e Cultura dell' Arma del Genio (ISCAG), col fine più modesto di aprire il sipario sul tema della fortificazione costiera negli anni del primo conflitto mondiale e sul suo attuale stato di conservazione.

\section{Note}

${ }^{1}$ AUSSME, Comando del Corpo di Stato Maggiore, Scacchiere Orientale, Memorie riguardanti le fortificazioni austriache nella frontiera italiana,
nell'Istria e nella Dalmazia, v.w. Fortificazioni sulla costa Dalmata tra Pola e Cattaro, b. 10, fasc. 44.6.

2 AUSSME, Comando del Corpo di Stato Maggiore, Scacchiere orientale, Memorie riguardanti le fortificazioni austriache nella frontiera italiana, nell'Istria e nella Dalmazia, X. Fortificazioni di Cattaro, b. 10, fasc. 44.9 .

${ }^{3}$ AUSSME, Comando del Corpo di Stato Maggiore, Scacchiere orientale, Memorie riguardanti le fortificazioni austriache nella frontiera italiana, nell'Istria e nella Dalmazia, X. Fortificazioni di Cattaro, b. 10, fasc. 44.9.

${ }^{4}$ Studio del fronte di terra. Individuazione dei XVI Distretti. (1918). ISCAG, Comando Generale, Genio, Ordinamento (5), Piazza marittima di Pola.

${ }^{5}$ AUSSME, Comando del Corpo di Stato Maggiore, Scacchiere Orientale, Memorie riguardanti le fortificazioni austriache nella frontiera italiana, nell'Istria e nella Dalmazia, v.w. Fortificazioni sulla costa Dalmata tra Pola e Cattaro, b. 10, fasc. 44.6.

\section{Bibliography}

Bravetta, E. (1925). La grande guerra sul mare, Mondadori, Milano, vol. I.

Castagnolo, V.; De Cadilhac, R.; Perfido, P.; Rossi, G.; eds. (2017). Architettura fortificata. Rilievo e restauro, Aesei editore, Martina Franca.

Damiani, G.; Fiorino, D.R. (2017). Military Landscapes, Scenari per il futuro del patrimonio Militare, Skira Ed., Milano.

Fortificazione delle coste. (1894). Rivista di Artiglieria e Genio, III, Roma, pp. 215-272.

Fortificazioni di Pola (1884). Rivista di Artiglieria e Genio, III, Roma, pp. 520-525.

Fortificazioni in Austria. (1885). Rivista di Artiglieria e Genio, II, Roma, pp. 354-355.

Guidetti, A. (1913). La fortificazione permanente, Francesco Bertinati editore, Torino.

Marsetič, R. (2012). “Analisi dell’Apparato militare austro-ungarico a Pola durante la Prima guerra mondiale", Atti, XLII, pp. 483-520.

Ministero della Marina, Ufficio del Capo di Stato Maggiore. (1917). Monografia militare del Litorale Austro-Ungarico. Pola, fronte a mare, vol. II, parte I.

Righi, E. (1906). "Sull'Armamento delle Piazze Forti marittime”, Rivista di Artiglieria e Genio, vol. IV, Roma, pp. 186-213.

Rocchi, E. (1906). "La difesa costiera al principio del XX secolo", Rivista di Artiglieria e Genio, vol. II, Roma, pp. 177-197.

Von Leithner, E. (1895). Principi fondamentali della fortificazione permanente moderna, Rocchi, E., trad., Voghera Ed., Roma. 